\title{
Ex ante versus ex post equilibria in classical Bayesian games with a nonlocal resource
}

\author{
Mátyás Koniorczyk \\ Wigner Research Centre for Physics, H-1525 Budapest, P.O. box 49, Hungary and \\ Institute of Mathematics and Informatics, Faculty of Science, \\ University of Pécs, H-7624 Pécs, Ifjúság útja 6, Hungary \\ András Bodor \\ Institute of Mathematics and Informatics, Faculty of Science, \\ University of Pécs, H-7624 Pécs, Ifjúság útja 6, Hungary \\ Miklós Pintér \\ Institute of Mathematics, Budapest University of Technology and Economics, H-1111 Budapest, Egry József u. 1, Hungary
}

\begin{abstract}
We analyze the difference between ex ante and ex post equilibria in classical games played with the assistance of a nonlocal (quantum or no-signaling) resource. In physics, the playing of these games is known as performing bipartite Bell-type experiments. By analyzing the Clauser-Horn-ShimonyHolt game, we find a constructive procedure to find two-person Bayesian games with a nonlocal (i.e. no-signaling, and, in many cases, quantum) advantage. Most games of this kind known from the literature can be constructed along this principle, and share the property that their relevant ex ante equilibria are ex post equilibria as well. We introduce a new type of game, based on the Bell-theorem by Vértesi and Bene, which does not have the latter property: the ex ante and ex post equilibria differ.
\end{abstract}

\section{INTRODUCTION}

It is widely accepted that Bell-type experiments and nonlocality are deeply related to game theory. It is known that in a classical strategic game the use of nonlocal signals may have certain benefits, such as new, better Pareto equilibria (see e.g. Ref. [1]). Brunner and Linden 2] have pointed out the deep relation between Bell-inequalities and the theory of Bayesian games leading to a new insight in case of both problems. This line of research is not to be confused with "quantum game theory" 3], which is dealing with scenarios constructed from classical nonBayesian games by a specific quantization procedure and not involving quantum or other nonclassical correlations. That kind of quantization of a classical game leads to a modified classical game [4], while in our case the classical game is Bayesian and not quantized, but it is played with the aid of a nonlocal resource possibly implemented with quantum systems.

Nonlocal correlations can lead to new equilibria in classical Bayesian games. This has attracted significant research attention. The general notion of these equilibria was studied by Auletta et al. [5]. Brandenburger and La Mura 6] addressed the question of quantum improvement from the perspective of team decision problems. Meanwhile, there are many particular classical games known for having an improvement when quantum nonlocal resources can be used. Examples include the "prototype" CHSH game which is a game-type description of the Bell-CHSH inequality [7], the entanglement-assisted orientation in space by Brukner [8], or the high-frequency trading example described in Ref. [6] just to mention a few. The CHSH game also has a conflict of interest variant 9] which inspired detailed studies of games with a similar equilibrium structure [10 12].

These games are ad-hoc constructions; a general mathematical recipe leading systematically to similar games is yet to be explored, and so is a systematic approach to find a game which benefits from the violation of a known Bell-inequality. In the present contribution we deal with two-player Bayesian games having a quantum advantage in order to make steps towards this direction. The detailed examination of the CHSH game leads us to a generic construction of games of the above kind which covers the other games, and it can serve as a recipe to find additional games of this kind. This common route of many games with a quantum advantage has not been pointed out before.

Having a Bell-type inequality at hand, it can be instructive to examine how its left-hand side relates to our construction. Searching for the maximal value of the lefthand side of the Bell inequality corresponds to a situation termed as ex ante in Bayesian game theory, whereas our construction originates from the ex post situation. These two eventually coincide in the case of the games considered in the literature. Consequently, the difference between these has not yet been explored.

However, the two situations can indeed differ, thereby leading to games with a different equilibrium structure. Here we introduce such a game: studying the Bell-type inequality by Vértesi and Bene [13], we find one which does not follow our construction and thus has an equilibrium structure different from that of the other known games.

This paper is organized as follows. In Section [I] we summarize some concepts of game theory we shall use. Section III is devoted to concepts related to Bayesian games, and the relation of those games to Bell-type experiments. On this basis we describe the structure of 


\begin{tabular}{|r||c|c|}
\hline$a \downarrow b \rightarrow$ & 0 & 1 \\
\hline \hline 0 & $(2,1)$ & $(0,0)$ \\
1 & $(0,0)$ & $(1,2)$ \\
\hline \hline$a \downarrow b \rightarrow$ & 0 & 1 \\
\hline \hline 0 & $(1,1)$ & $(0,0)$ \\
1 & $(0,0)$ & $(1,1)$ \\
\hline
\end{tabular}

TABLE I. The Battle of sexes game (left) and the coordination game (right). The columns and rows refer to the players' actions $a \in A, b \in B$, whereas the numbers in the tables are the payoffs in the format $\left(u_{\mathrm{A}}(a, b), u_{\mathrm{B}}(a, b)\right)$.

many Bayesian games related to Bell-type experiments from the point of view of their equilibria, and we describe a means for constructively defining such games. In Section IV we study the Vértesi-Bene Bell-inequality to show that it leads to a game with a different equilibrium structure. In Section $\mathrm{V}$ our results are summarized and conclusions are drawn.

\section{GAME THEORY OVERVIEW}

Let us start with a brief review of some concepts of game theory; we refer to Ref. [14] for their detailed description. Games in strategic form are common models in game theory. In case of two players and finite strategy sets, they are also termed as bimatrix games. A bimatrix game is a quadruple $\left(A, B, u_{\mathrm{A}}, u_{\mathrm{B}}\right)$, where $A$ and $B$ are finite sets representing the set of actions of the two players, Alice and Bob, respectively, whereas $u_{\mathrm{A}}$ and $u_{\mathrm{B}}$ are $A \times B \mapsto \mathbb{R}$ functions representing the payoff (or utility) of each player. Throughout this paper we shall, without loss of generality, label the actions with numbers.

A central concept of game theory is that of the equilibrium. The (pure) Nash equilibrium of a game [15] is a selection of actions so that it is not worth deviating from the given strategy by any of the players unilaterally: $(a, b)$ is a Nash equilibrium if

$$
\begin{gathered}
\left(\forall a^{\prime} \in A\right) \quad u_{\mathrm{A}}\left(a^{\prime}, b\right) \leq u_{\mathrm{A}}(a, b), \\
\left(\forall b^{\prime} \in B\right) \quad u_{\mathrm{B}}\left(a, b^{\prime}\right) \leq u_{\mathrm{B}}(a, b) .
\end{gathered}
$$

As an example consider the game called "battle of sexes", defined in the left of Tab. [1. (The common story behind it is that Alice prefers going to the cinema whereas Bob prefers going to a soccer match, but they would like to be together.) The game has two pure equilibria, $a=b=0$ and $a=b=1$.

An extension to the concept of strategies is to consider probability distributions over the strategies, socalled mixed strategies. Having $p_{\mathrm{A}}(a)$ and $p_{\mathrm{B}}(b)$, two independent probability distributions on Alice's and Bob's side, one can calculate the expected value of the utility, the expected payoff using the joint distribution being the product of those on the two sides. The probability distributions are termed as mixed strategies as opposed to the deterministic pure strategies mentioned so far. The concept of the equilibrium can be extended in a straightforward manner by considering mixed strategies and the expected payoff. According to the celebrated theorem by Nash [15, 16], a mixed equilibrium always exists in the games studied here. In the case of the "battle of sexes", in addition to the aforementioned pure equilibria, there is also a mixed one with the probability distributions $p_{A}=(2 / 3,1 / 3)$ and $p_{B}=(1 / 3,2 / 3)$, resulting in the expected payoff of $(2 / 3,2 / 3)$.

Finally, an even more general equilibrium concept is that of correlated equilibrium [17, in which we allow for a joint probability distribution $p(a, b)$ to determine the choice of the players. Its definition reads

$$
\begin{aligned}
& \left(\forall a, a^{\prime}\right) \sum_{b}\left(u_{\mathrm{A}}(a, b)-u_{\mathrm{A}}\left(a^{\prime}, b\right)\right) p(a, b) \geq 0 \\
& \left(\forall b, b^{\prime}\right) \sum_{a}\left(u_{\mathrm{B}}(a, b)-u_{\mathrm{B}}\left(a, b^{\prime}\right)\right) p(a, b) \geq 0 .
\end{aligned}
$$

From amongst the mentioned equilibrium concepts, this is maybe the easiest to tackle mathematically as the equilibrium strategies form a polytope in the set of probability distributions. If, however, this distribution happens to be correlated, then in order to implement the strategy the players may have to rely on the advice of a trusted coordinator or they have to agree in advance on the strategies. An example of such an equilibrium appears e.g. in the "coordination game", defined in the right of Tab. I Here $p(0,0)=p(1,1)=1 / 2$ is a correlated equilibrium (there are infinitely many other correlated equilibria though). Coordination games frequently appear in quantum-assisted situations. The equilibrium in the correlated case takes the intuitive meaning that it is not worth deviating from the advice of the trusted coordinator unilaterally.

\section{BAYESIAN GAMES AND BELL-TYPE EXPERIMENTS}

It is prevalently accepted that a Bell-type experiment can also be interpreted as a kind of game termed as "Bayesian game" in the language of game theory. In such games, Alice and Bob choose some input first (or get it from a third party). In physics we say that both Alice and Bob choose a measurement, whereas in the game theory language, they become aware of their types $x \in X$ and $y \in Y$ first ( $X$ and $Y$ are finite sets). Then they choose an action, and the payoff depends on both the types and the actions, so we have $u_{\mathrm{A}}(a, b, x, y)$ and $u_{\mathrm{B}}(a, b, x, y)$, respectively, thus we have a strategic form game for each pair of types $(x, y)$. (Note that in many applications of Bayesian games the payoff does not depend on the other player's type.) In the Bell-type experimental scenario the actions are the results $a \in A(b \in B)$ of the measurement $x \in X(y \in Y)$ the latter playing the role of the types. They are assumed to be chosen by the parties independently at will; this choice can thus be modeled as a 


\begin{tabular}{|lll||cc|cc|}
\hline \multicolumn{2}{|c||}{$\begin{array}{l}x \downarrow \\
\end{array}$} & $a \downarrow$ & \multicolumn{2}{c}{0} & \multicolumn{2}{c|}{1} \\
& 0 & 0 & 1 & 0 & 1 \\
\hline \hline 0 & 0 & 1 & -1 & 1 & -1 \\
& 1 & -1 & 1 & -1 & 1 \\
\hline \multirow{2}{*}{1} & 0 & 1 & -1 & -1 & 1 \\
& 1 & -1 & 1 & 1 & -1 \\
\hline
\end{tabular}

\begin{tabular}{|c|c|c|c|c|c|}
\hline$x \downarrow$ & $y \rightarrow$ & & & 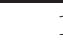 & \\
\hline & $a \downarrow \quad b \rightarrow$ & 0 & 1 & 0 & 1 \\
\hline 0 & 0 & $1 / 2$ & 0 & $1 / 2$ & 0 \\
\hline & 1 & 0 & $1 / 2$ & 0 & $1 / 2$ \\
\hline 1 & 0 & $1 / 2$ & 0 & 0 & $1 / 2$ \\
\hline & 1 & 0 & $1 / 2$ & $1 / 2$ & 0 \\
\hline
\end{tabular}

TABLE II. The payoff function of the CHSH game (top) and the PR-box, the no-signaling behavior maximizing it (bottom). The tabular divides into blocks indexed with types. Within each pair of types, we have a game payoff matrix similar to that in [. There is a single payoff value in each entry as the payoffs are equal for both parties.

probability distribution of their possible types (aka measurements). In order to verify e.g. the violation of local realism, some expression of the outcomes (aka actions) is evaluated.

As of the equilibria of Bayesian games, there are three situations studied in game theory. In the ex ante situation, no players know each others' types and neither that of their own, thus regarding equilibria, the relevant payoffs are expected values on some prior distribution of all types. The interim case is when each player knows his own type but has only a probability distribution on that of the others'. Hence, when the payoff is studied, each player considers the expected value on the others' types. It is prevalently known in game theory that in the case of the games studied here the set of ex ante and interim equilibria coincide. Finally, in an ex post situation, all players know everyone's types, thus we fall back to the case of simple strategic form games. So for a given $(x, y)$, we can consider the equilibrium properties of the game defined by the actions and the respective payoffs $u_{\mathrm{A}}(a, b, x, y), u_{\mathrm{A}}(a, b, x, y)$ with $x$ and $y$ fixed. Hence, the set of strategies are classified by the conditional probability distributions $P(a, b \mid x, y)$ which describe the behavior of the nonlocal resource in a Bell-type scenario.

Consider, for instance, the case of the CHSH experiment (CHSH game). The payoff is the same for both players: $u_{\mathrm{A}}=u_{\mathrm{B}}=u$; it is tabulated on the upper part of Tab II

The expected payoff for such a game, given a uniform prior on the types $(x, y)$ reads

$$
U=\frac{1}{4} \sum_{x, y} \sum_{a, b} u(a, b, x, y) P(a, b \mid x, y),
$$

thus it is linear in the conditional probability character- izing the behavior. Based on the assumption on the behaviors as constraints of its maximization, it can attain various maxima.

A possible assumption is locality: the conditional probabilities are the convex combinations of products of deterministic local channels (i.e. which assign a set of pure strategies to each type). Hence, they form a polyhedron in the vector space of conditional probabilities $P(a, b \mid x, y)$, which is thus naturally given in its vertex representation, that is, as a convex combination of its aforementioned vertices [18, 19]. This polytope is termed as the local polytope $\mathcal{P}_{\mathrm{L}}$. The maximization of the payoff in (3) subject to $P(a, b \mid x, y) \in \mathcal{P}_{\mathrm{L}}$ is a linear program. The CHSH inequality's RHS is this local maximum, which is $1 / 2$ in this case. (Recall that we calculate the ex ante expected payoffs with a uniform prior on the types. In the classic literature of the Bell-CHSH inequality, the prevalently known local bound is 2 , which is the ex-post value, hence our bounds are to be multiplied by 4 to achieve the usual values.)

A broader class of behaviors is that of the quantum ones. The set of quantum behaviors in the space of conditional probabilities is a convex domain which is a superset of $\mathcal{P}_{\mathrm{L}}$, and can be characterized by an infinite series of semidefinite programs [20, 21]. The heuristically determined maximal value is $\sqrt{2} / 2$ known as Tsirelson's bound [22].

Finally, the broadest set frequently considered is that of the no-signaling behaviors [19]. In their case the single assumption is that the given resource cannot be used for transmitting any classical information between the two parties. Mathematically this can be formulated as the no-signaling conditions:

$$
\begin{aligned}
& \forall a, x, y_{1}, y_{2} \quad \sum_{b} P\left(a, b \mid x, y_{1}\right)=\sum_{b} P\left(a, b \mid x, y_{2}\right), \\
& \forall b, x_{1}, x_{2}, y \quad \sum_{a} P\left(a, b \mid x_{1}, y\right)=\sum_{a} P\left(a, b \mid x_{2}, y\right),
\end{aligned}
$$

defining a polytope $\mathcal{P}_{\text {NS }}$ in the space of conditional probabilities again. Solving the linear program of finding the maximal payoff in (3) subject to $P(a, b \mid x, y) \in \mathcal{P}_{\mathrm{NS}}$ leads to the objective value of 1 . (This corresponds to the ex-post value of 4 which is known in the Bell-inequality literature.) As in case of all such linear programs, it is obtained at a vertex of $\mathcal{P}_{\mathrm{NS}}$, outside $\mathcal{P}_{\mathrm{L}}$ and also outside the quantum domain. We tabulate this behavior in the lower part of Tab. III. The fictitious device realizing this is the Popescu-Rorhlich (PR) box [23 26].

Let us now make two important observations. Firstly, as the payoffs in these games are equal, maximizing the expected payoff necessarily leads to an ex ante equilibrium. Were this not the case, it would be possible for at least one player to unilaterally deviate, thereby obtaining a better payoff. As the payoff of the other party is the same, a better payoff could be obtained which would be higher than the maximum, which is impossible. Secondly, recall that if once $\left(x_{0}, y_{0}\right)$ is fixed, in the ex post situation the payoff depends only on the actions, and 
hence the block $P\left(a, b \mid x_{0}, y_{0}\right)$ of the conditional distribution becomes a (possibly correlated) strategy profile of the game corresponding to $\left(x_{0}, y_{0}\right)$. Hence, given such a distribution it can be verified whether it is an equilibrium using the definitions of equilibrium of bimatrix games. In the case of the CHSH game, given any particular pair of types $(x, y)$, the respective block of the optimal no-signaling behavior is an equilibrium of the game. Otherwise speaking, an ex post equilibrium is achieved. A possible intuitive interpretation is the following. A crucial assumption of the Bell-situations is that none of the parties is aware of the other's type. And indeed, we are using a PR-box which is a no-signaling resource. However, our observation implies that it is not worth for any of the players to deviate from the advice of the box, even if they would become aware of the other's type which is the ex post situation. A direct calculation shows that the same holds for the optimal quantum behavior, as it is a convex combination of the PR-box and the uniform distribution in all blocks.

Studying further the construction of the CHSH game another observation can be made. If we were to search for a game which is interesting from the point of view of nonlocal resources and we wouldn't be aware of this one, it would be possible to construct it as follows. Pick a nonlocal vertex of the no-signaling polytope $\mathcal{P}_{\mathrm{NS}}$, this defines a behavior. Then define the payoff matrix of a Bayesian game so that for all the blocks for given types $(x, y)$, the corresponding probability distribution is its correlated equilibrium according to Eq. (2). For a fixed behavior $p(a, b)$, Eq. (2) defines a nonempty polyhedron in the space of payoff matrices, so there may be infinitely many choices. In addition, the payoff should be chosen such a way that the maximum of the expected payoff for a selected prior (e.g. the uniform distribution of types) over the local polytope $\mathcal{P}_{\mathrm{L}}$ is lower than the no-signaling value on the chosen vertex. In our particular example, the CHSH game could be constructed entirely from the vertex: the PR-box, which is the only nontrivial nonlocal no-signaling vertex in the two-input-two-output case. The CHSH game can be obtained along the above lines. As the expected payoff is a linear function, and the quantum domain is a convex one between $\mathcal{P}_{\mathrm{L}}$ and $\mathcal{P}_{\mathrm{NS}}$, it is likely that there will be a quantum advantage, too.

All the two-player games with a quantum advantage we are aware of derive from this construction. The examples include the high-speed trading example by Brandenburger and La Mura [6] and the spatial orientation game by Bruckner [8], two games found in a heuristic way independently from each other. These two different payoffs can be derived from the same vertex. The "conflict of interest game" in Ref. [9], even though it is not directly a coordination game, also obeys this kind of construction, using the same vertex as the CHSH game.

\begin{tabular}{|c|c|c|c|c|c|}
\hline \multirow{2}{*}{$x \downarrow$} & \multirow{2}{*}{$\begin{array}{l}y \rightarrow \\
a \downarrow \quad b \rightarrow\end{array}$} & \multicolumn{2}{|c|}{0} & \multicolumn{2}{|c|}{1} \\
\hline & & 0 & 1 & 0 & 1 \\
\hline \multirow{2}{*}{0} & 0 & 0 & $-c / 2$ & $\begin{array}{lc}c / 2 \\
\end{array}$ & $-c / 2$ \\
\hline & 1 & $-c / 2$ & 0 & 0 & 0 \\
\hline \multirow{2}{*}{1} & 0 & $c / 2$ & 0 & $-c$ & 0 \\
\hline & 1 & $-c / 2$ & 0 & 0 & 0 \\
\hline \multirow{3}{*}{2} & 0 & $1 / 2$ & $-1 / 2$ & $1 / 2$ & $-1 / 2$ \\
\hline & 1 & $\frac{\sqrt{2}+2}{4}$ & $\frac{\sqrt{2}-2}{4}$ & $\frac{\sqrt{2}-6}{4}$ & $\frac{\sqrt{2}-2}{4}$ \\
\hline & 2 & 0 & 0 & 0 & 0 \\
\hline
\end{tabular}

TABLE III. The payoff of the Vértesi-Bene game in Ref. [13], $c>0$ is a parameter.

\begin{tabular}{|c|c|c|c|c|}
\hline & $y \rightarrow$ & 0 & & 1 \\
\hline & $a \downarrow \quad b \rightarrow$ & 0 & 0 & $\begin{array}{ll}0 & 1\end{array}$ \\
\hline 0 & 0 & 1 & 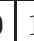 & 10 \\
\hline & 1 & 0 & 0 & $\begin{array}{ll}0 & 0\end{array}$ \\
\hline 1 & 0 & 1 & 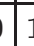 & 10 \\
\hline & 1 & 0 & & 00 \\
\hline & 0 & 1 & & 10 \\
\hline 2 & 1 & 0 & & $\begin{array}{ll}0 & 0\end{array}$ \\
\hline & 2 & 0 & & $\begin{array}{ll}0 & 0\end{array}$ \\
\hline
\end{tabular}

TABLE IV. An optimal local behavior for the game in Table III The blocks in boldface are not equilibria of the game in the corresponding block.

\section{AN EXCEPTION: THE VÉRTESI-BENE GAME}

The question naturally arises whether there are examples of Bayesian games with a nonlocal advantage which do not obey our construction, and have a different equilibrium structure. In search for such an exception, let us investigate the bipartite Bell-situation which was studied by Vértesi and Bene in Ref. [13] which was derived to prove the possible nontrivial role of POVM measurements in Bell-type scenarios. A peculiar feature of this case is that Alice has 3 inputs, and only for the third input there are 3 outputs. In case of the first two inputs, the number of outputs is 2. Bob has two inputs and two outputs for both. The payoff function is tabulated in Table III with a parameter $c>0$. This attains a local bound of 1 , e.g. by the vertex of the local poytope tabulated in Table IV] apparently regardless of the value of $c$. Note that the blocks typeset in bold are not equilibria of the respective game (the bimatrix one defined by the same block of the payoff), thus clearly the local vertex maximizing the expected payoff belongs to a non-equilibrium behavior.

Let us now leave the local polytope and check the nosignaling case. It is the linear programming problem of 


\begin{tabular}{|c|c|c|c|c|c|}
\hline \multirow[t]{2}{*}{$x \checkmark$} & \multirow{2}{*}{$\begin{array}{l}y \rightarrow \\
a \downarrow b \rightarrow\end{array}$} & \multicolumn{2}{|c|}{0} & \multicolumn{2}{|c|}{1} \\
\hline & & 0 & 1 & 0 & 1 \\
\hline \multirow{2}{*}{0} & 0 & $1 / 2$ & 0 & $1 / 2$ & 0 \\
\hline & 1 & 0 & $1 / 2$ & 0 & $1 / 2$ \\
\hline \multirow{2}{*}{1} & 0 & $1 / 2$ & 0 & 0 & $1 / 2$ \\
\hline & 1 & 0 & $1 / 2$ & $1 / 2$ & 0 \\
\hline \multirow{3}{*}{2} & 0 & $1 / 2$ & 0 & $1 / 2$ & 0 \\
\hline & 1 & 0 & 0 & 0 & 0 \\
\hline & 2 & 0 & $1 / 2$ & 0 & $1 / 2$ \\
\hline
\end{tabular}

TABLE V. The optimal no-signaling behavior for the game in Tab. III for $c>1$. The block in boldface is not an equilibrium of the game in the corresponding block.

finding the maximum of average payoff assuming an uniform prior on the types. This is the objective function, and the feasibility region is the local polytope $\mathcal{P}_{\mathrm{L}}$, defined by the trivial normalization of the behaviors and the no-signaling conditions in Eq. (4). For $c>1$, the optimum is achieved at a vertex which corresponds to the nonlocal behavior tabulated in Tab. $\nabla$ Observe that the optimal no-signaling vertex has a block (in particular, the one belonging to Alice's third type and Bob's first type), which is not an equilibrium of the game. So in this case the ex ante and ex post equilibria differ. In the case of other games like the CHSH the presence of an optimal no-signaling box can assist in the realization of an equilibrium which could not be further improved by letting the players to have full information of the others' type. In contrast to that, in the just studied game the players could further benefit from actual information on the other's type, albeit the nonlocal box also leads to an improvement over the classical result. Hence, there are games that could be played better if the players wouldn't be restricted to no-signaling resources but were aware of the other's type. Otherwise speaking, if Alice and Bob knew each other's type with certainty (e.g. via some communication channel), they would play differently from having just the no-signaling box at hand. This is a feature not present in the other known two-player games.

The question naturally arises whether this game has similar features when a quantum box is used by the players. Ref. [13] contains an example of a quantum behavior violating the classical bound in this case (not an extremal one though). This is tabulated in Tab. VI, In the case of the first two types of Alice, the CHSH case is repeated by the construction in Ref. [13], whereas in the lowest two blocks we have evaluated their numerical construction. Interestingly, now both of the blocks for Alice's type 2 (the bold-typed ones) are non-equilibria, thus this behavior is not an ex post equilibrium. It can be verified via direct calculation that it is an ex ante equilibrium meanwhile.

\begin{tabular}{|c|c|c|c|c|c|}
\hline & \multirow{2}{*}{$\begin{array}{l}y \rightarrow \\
a \downarrow \quad b \rightarrow\end{array}$} & \multicolumn{2}{|c|}{0} & \multicolumn{2}{|c|}{1} \\
\hline & & 0 & 1 & 0 & 1 \\
\hline \multirow{2}{*}{0} & " 0 & $\frac{2+\sqrt{2}}{8}$ & $\frac{2-\sqrt{2}}{8}$ & $\frac{2+\sqrt{2}}{8}$ & $\frac{2-\sqrt{2}}{8}$ \\
\hline & 1 & $\frac{2-\sqrt{2}}{8}$ & $\frac{2+\sqrt{2}}{8}$ & $\frac{2-\sqrt{2}}{8}$ & $\frac{2+\sqrt{2}}{8}$ \\
\hline \multirow[b]{2}{*}{1} & 0 & $\frac{2+\sqrt{2}}{8}$ & $\frac{2-\sqrt{2}}{8}$ & $\frac{2-\sqrt{2}}{8}$ & $\frac{2+\sqrt{2}}{8}$ \\
\hline & 1 & $\frac{2-\sqrt{2}}{8}$ & $\frac{2+\sqrt{2}}{8}$ & $\begin{array}{r}8 \\
\frac{2+\sqrt{2}}{8} \\
\end{array}$ & $\begin{array}{c}8 \\
\frac{2-\sqrt{2}}{8}\end{array}$ \\
\hline \multirow{3}{*}{2} & 0 & 0.30602 & 0.12925 & 0.41652 & 0.01875 \\
\hline & 1 & 0.18243 & 0.11444 & 0.00395 & 0.29293 \\
\hline & 2 & 0.01155 & 0.25630 & 0.07953 & 0.18832 \\
\hline
\end{tabular}

TABLE VI. The quantum behavior with an advantage discussed in Ref. [13]. The values have been obtained by the direct evaluatinon of the result in the cited paper. The blocks in boldface are not equilibria of the game in the corresponding block of Tab III

\section{CONCLUSIONS}

In conclusion, we have studied two-player classical Bayesian games played with the assistance of a nonlocal resource. These correspond to bipartite Bell-type experiments in physics. We have addressed the interpretation of these situations in different phases of the game, depending on what is revealed for the players. We have found that the ex post equilibria and the ex ante ones achievable with no-signaling or quantum resources coincide in the games studied in the literature. In contrast to that, we have found a game in which these equilibria are different.

It would be interesting to find some intuitive explanation of this unusual behavior of the game. It is likely that it relates to the high asymmetry of the given situation. This also reduces the symmetries of the relevant polytopes, which is necessary for the corresponding Bell theorem to prove that POVM-s can have a nontrivial role in the theory of quantum information. What we found here is that this Bell-situation, in addition to accomplishing this goal, also exhibits an interesting game-theoretic structure, which can be a subject of further research.

Refs. [1, [5] define the notion of a variety of equilibrium concepts of Bayesian games played in the presence of local resources, thereby paving the way to the study of the equilibrium structure of these games. In the present paper we find a generic construction for these kinds of games, and reveal a particular property of the equilibria which is not observable in every game.

\section{ACKNOWLEDGMENTS}

The authors acknowledge the support of the National Research, Development and Innovation Office (NKFIH) under the contracts Nos. K124631 and K124351. M.P. thanks GAMENET for support. We thank Gernot Alber and Tamás Kiss for useful discussions. A. B. wants to 
express special thanks to Tamás Geszti.

[1] P. La Mura, International Journal of Quantum Information 3[18\$3T(2065t)esi and E. Bene, Phys. Rev. A 82, 062115 (2010).

[2] N. Brunner and N. Linden, [14] M. Maschler, E. Solan, and S. Zamir, Game Theory Nature Commun. 4, 2057 (2013).

[3] J. Eisert, M. Wilkens, and M. Lewenstein, Phys. Rev. Lett. 83, 3077 (1999)

[4] S. J. van Enk and R. Pike, Phys. Rev. A 66, 024306 (2002)

[5] V. Auletta, D. Ferraioli, A. Rai, G. Scarpa, and A. Winter, CEUR Workshop Proceedings 1949, 198 (2017), arXiv:1605.07896v3.

[6] A. Brandenburger and P. L. Mura, Phil. Trans. R. Soc. A 374, 20150096 (2016)

[7] J. F. Clauser, M. A. Horne, A. Shimony, and R. A. Holt, Phys. Rev. Lett. 23, 880 (1969).

[8] C. Brukner, N. Paunković, T. Rudolph, and V. Vedral, International Journal of Quantum Information 04, 365

[9] A. Pappa, N. Kumar, T. Lawson, M. Santha, S. Zhang, E. Diamanti, and I. Kerenidis, Phys. Rev. Lett. 114, 020401 (2015).

[10] A. Rai and G. Paul, Phys. Rev. A 96, 042340 (2017)

[11] A. Roy, A. Mukherjee, T. Guha, S. Ghosh, S. S. Bhattacharya, and M. Banik, Phys. Rev. A 94, 032120 (2016)

[12] K. Bolonek-Lasoń, Int. J. of Quant. Inf. 15, 1750033 (2017)

(Cambridge University Press, Cambridge, 2013).

[15] J. Nash, Proceedings of the National Academy of Sciences 36, 48 (1950).

[16] J. Nash, The Annals of Mathematics 54, 286 (1951).

[17] R. J. Aumann, Journal of Mathematical Economics 1, 67 (1974)

[18] J. Wilms, Y. Disser, G. Alber, and I. C. Percival, Phys. Rev. A 78, 032116 (2008).

[19] N. Brunner, D. Cavalcanti, S. Pironio, V. Scarani, and S. Wehner, Rev. Mod. Phys. 86, 419 (2014)

[20] M. Navascués, S. Pironio, and A. Acín, Phys. Rev. Lett. 98, 010401 (2007).

[21] M. Navascués, S. Pironio, and A. Acín, New J. Phys. 10, 073013 (2008).

606[22] B. S. Cirel'son, Lett. Math. Phys. 4, 93 (1980)

[23] P. Rastall, Found. Phys. 15, 963 (1985).

[24] L. Khalfin and B. Tsirelson, in Symposium on the Foundations of Modern Physics, edited by P. Lahti and P. Mittelstaedt (World. Sci. Publ., 1985) pp. 441-460.

[25] S. Popescu and D. Rohrlich, Phys. Lett. A 166, 293 (1992)

[26] S. Popescu and

Found. Phys. 24, 379 (1994).

D. Rohrlich, 